\title{
Nueva espeCie del género SiCyOs L. \\ (Cucurbitaceae) para la República Mexicana
}

\author{
Isela Rodríguez-Arévalo y Rafael LiRa SaAde \\ Laboratorio de Recursos Naturales, Unidad de Biotecnología y Prototipos, FES Iztacala \\ Universidad Nacional Autónoma de México. \\ Avenida de los Barrios s.n., Los Reyes Iztacala, Tlalnepantla, C.P. 54090, Estado de México. \\ Tel. (5)6 2311 37; Fax (5)6 2312 25; Email: gaia2000@prodigy.net.mx; rlira@servidor.unam.mx
}

\begin{abstract}
Resumen. Se describe y se ilustra una nueva especie del género Sicyos ( $S$. daviliae) para los estados de Chiapas y Oaxaca en el sur de México. Esta nueva especie se distingue del resto de las especies mexicanas con frutos secos y espinosos del género, principalmente por la coloración de sus frutos, los cuales en fresco son verde hialinos y ocasionalmente maculados en color negro. Es similar a Sicyos warmingii Cogn. de Brasil y Argentina, de la cual difiere en varias características tales como la consistencia de la lámina, las ramificaciones de los zarcillos, la forma del estigma, y la coloración y tamaño de sus frutos.

Palabras clave: Cucurbitaceae, México, Sicyos, Sicyos daviliae, Sicyos warmingii.

Abstract. A new species of Sicyos ( $S$. daviliae) from the southern Mexican states of Chiapas and Oaxaca is described and ilustrated. This new species is distinguished from the remaining Mexican species of Sicyos with dry and spinous fruits, mainly by the color of its fruits, which when fresh are green-hyaline and occassionally black-maculated. It is similar to Sicyos warmingii Cogn., from Brasil and Argentina, from which differs in several features such as blade consistency, tendril branches, stigma shape and fruit color and size.
\end{abstract}

Key words: Cucurbitaceae, México, Sicyos, Sicyos daviliae, Sicyos warmingii.

$\mathrm{E}$ 1 género Sicyos, propuesto por Linneo en 1753 para ubicar a tres especies ( $S$. angulata, S. laciniata y S. trifoliata), pertenece a la tribu Sicyae, subtribu Sicyinae, y se considera uno de los más diversos de la familia Cucurbitaceae (Jeffrey, 1990). Como suele ocurrir con muchos otros taxa vegetales, México es el centro de diversificación más importante para el género, ya que dentro de su territorio prosperan al menos 19 especies (Rodríguez-Arévalo, 2001). Una de esas 19 especies es la que aquí se propone como nueva para la ciencia.

Sicyos daviliae Rodríguez-Arévalo et Lira, sp. nov. (figura 1)

S. warmingii Cogn. similis, sed differt laminis foliorum papyraceis, capreolis 3-4 ramis, stigmate 3-lobo, lobis rotundatis vel rhombiformibus, fructibus semper armatis, viridi-hyalinis, aliquibus maculis nigris.

TIPO: México, Chiapas. Mpio. Motozintla de Mendoza. A 2 km de la entrada a Berriozabal. $15^{\circ} 19.395^{\prime} \mathrm{N}$; $92^{\circ} 17.381$ 'W. Alt. $1708 \mathrm{~m}$. Bosque mesófilo de montaña. 9/11/1999. I. Rodríguez \& J.I. Calzada 287. (hojas, flores estaminadas, flores pistiladas y frutos. (Holotipo: MEXU; Isotipos: IZTA, MICH).

Plantas herbáceas, rastreras y trepadoras, aparentemente anuales. Tallos delgados, ramificados, sulcados, glabros a escasamente pubescente-vellosos, principalmente en los nudos. Hojas sobre pecíolos delgados, 0.4-15.8 cm de largo, sulcados, escasa a densamente pubescente-vellosos; lámina papirácea, $3.0-14.5 \mathrm{~cm}$ de largo, 3.4-16.3 cm de ancho, anchamente ovado-triangular, ligera a profundamente 5-7 lobada, los lóbulos anchos, triangulares, el central fuertemente acuminado y más largo que los laterales, la lámina nunca envolviendo con su superficie abaxial a las flores pistiladas y/o los frutos de la misma axila, margen ligera a fuertemente serrulado-denticulado, ambas superficies con tricomas cónicos, ligeramente recurvados, en la abaxial mas abundantes, de menor tamaño y las venas más resaltadas. Zarcillos con pedúnculos delgados, 0.9-6.9 cm de largo, sulcados, 3-4 ramifica- 
dos, glabros a escasamente pubescentes a vellosos. Flores estaminadas dispuestas en panículas conspicuas, 4.2-28.7 cm de largo, siempre más largas que la hoja adyacente, pedunculadas, el pedúnculo $3.1-23.1 \mathrm{~cm}$ de largo; raquis delgado, escasa a densamente pubescente-velloso, principalmente hacia el ápice; pedicelos delgados, 5.0-26.0 mm de largo, ocasionalmente reflexos, glabros a densamente pubescente-vellosos, principalmente hacia la base; receptáculo campanulado, 0.9-1.6 mm de largo, 2.5-3.6 mm de ancho; sépalos subulados a filiformes $0.4-1.0(-5) \mathrm{mm}$ de largo; pétalos 5-6, 1.5.3.6 mm de largo, 1.2-2.9 $\mathrm{mm}$ de ancho, triangulares, blanco a blanco-verdosos, superficie interna cubierta por tricomas pedunculados de ápice globoso, posiblemente glandulares, la externa glabra a densamente pubescente-vellosa; columna estaminal 2.1-3.0 de largo, nunca conspicuamente exerta, anteras completamente fusionadas formando una estructura globosa, tecas completamente arqueadas. Flores pistiladas numerosas, dispuestas en glomérulos, creciendo sobre un pedúnculo común, $0.5-2.5 \mathrm{~cm}$ de largo, extendiéndose hasta 1.5-6.0 cm en los frutos, escasa a densamente pubescente-velloso principalmente hacia la base; ovario 1-locular, ovoide, armado, densamente glandular-velloso; receptáculo y perianto como en las estaminadas, solo que más reducido y con 3-4 pétalos; columna de estilo y estigma 1.8-2.2 $\mathrm{mm}$ de largo, estigma 3 lobado, lóbulos redondeados a dilatado-romboides, reflexos, óvulo 1 en posición vertical. Frutos secos, numerosos, triangular-ovoides, $0.7-1.2 \mathrm{~cm}$ de largo, $0.4-0.8 \mathrm{~cm}$ de diámetro, armados, pared del fruto delgada, fuertemente adherida a la semilla, glabra a densamente pubescentevellosa, cuando fresca de color verde hialino con máculas negras, tornándose pardo clara a negra al secar; espinas hasta 11, 3.9-5.0 $\mathrm{mm}$ de largo, de base ancha, creciendo sobre pequeñas crestas hacia la mitad basal, aciculares, antrorsas, persistentes, retrobarba- das, excepto hacia la base, del mismo color que el resto del fruto. Semilla ovoide, pardo obscura.

Distribución y ecología. Esta especie sólo se ha recolectado en unas cuantas localidades de los estados de Chiapas y Oaxaca. Crece en vegetación secundaria de bosque mesófilo de montaña y ecotono de éste con encinar, así como en cafetales con Inga sapindiodes y milpas, en elevaciones entre 1300 y 2059 msnm. Florece y fructifica de julio a diciembre.

Nombres comunes. En Oaxaca se le conoce como chayotillo, un nombre que frecuentemente se asigna en México a las especies de Sicyos.

\section{Discusión}

Aunque muchas de las características de sus estructuras vegetativas y reproductivas son compartidas con otras especies mexicanas del género y algunas con Sicyos warmingii Cogn. de Brasil y Argentina (Martínez-Crovetto, 1964) (cuadro 1), la coloración y otros rasgos de sus frutos la hacen única y muy distintiva dentro del género. El nombre de esta especie es en reconocimiento a la Dra. Patricia Dávila Aranda (FESIztacala, UNAM), sin duda una de las más importantes e influyentes botánicas de México.

Ejemplares adicionales examinados. MEXICO. Chiapas. Mpio. Motozintla de Mendoza. Senda a Plan Grande, aproximadamente $200 \mathrm{~m}$ arriba del vivero. $15^{\circ} 20.578^{\prime} \mathrm{N} ; 92^{\circ} 15.021^{\prime} W$. Alt. 1522 m. 8/11/1999. I. Rodríguez \& J.I. Calzada 286. (hojas, flores estaminadas, flores pistiladas y frutos). (MEXU); Mpio. Motozintla de Mendoza. Senda a Plan Grande, arriba del vivero. $15^{\circ} 20.578^{\prime} \mathrm{N}$; $92^{\circ} 15.021^{\prime} \mathrm{W}$. Alt. $1522 \mathrm{~m}$. 8/11/1999. I. Rodríguez \& J.I. Calzada 286a. (frutos). (MEXU); Mpio. Motozintla de Mendoza. A 3.8

Cuadro 1. Comparación de las características de $S$. daviliae y S. warmingii.

Sicyos daviliae

Lámina papirácea de $3.0-14.5 \mathrm{~cm}$ de largo y $3.4-16.3 \mathrm{~cm}$ de ancho

\section{Zarcillos 3-4 ramificados}

Estigma trilobado, lóbulos redondeados a dilatado-romboides

Frutos de 7-12 mm de largo y de 4-8 mm de diámetro, verde hialino frecuentemente con algunas máculas negras, siempre armados
Sicyos warmingii

Lámina membranácea de $8.0 \mathrm{~cm}$ de largo y $20.0 \mathrm{~cm}$ de ancho

Zarcillos 4-5 ramificados a veces $2-3$

Estigma sub-3-lobado

Frutos de 8-12 mm de largo y de 4-4.5 mm de diámetro, verde-amarillento, ocasionalmente subinermes 


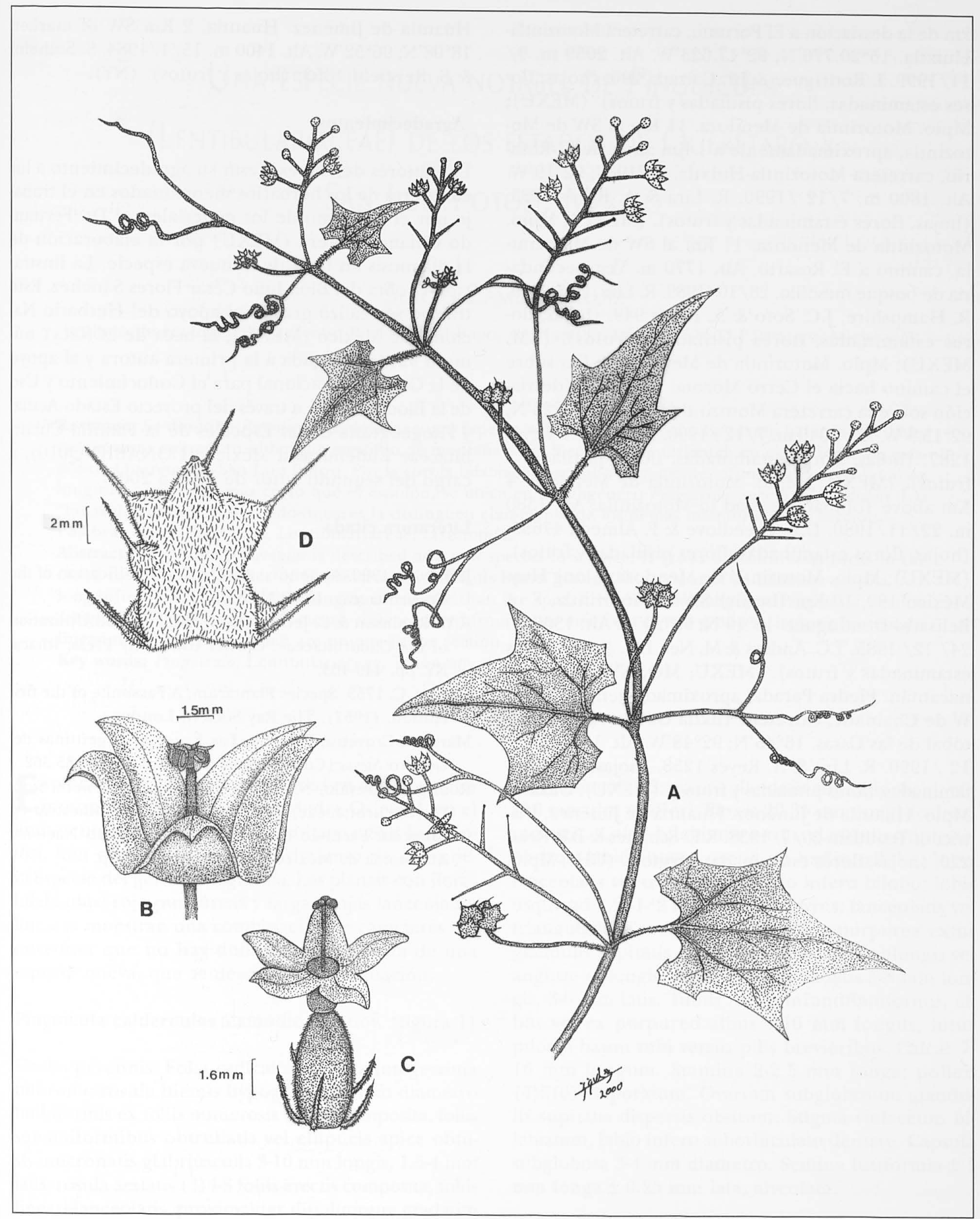

Figura 1. Sicyos daviliae Rodríguez-Arévalo et Lira. A) Hábito, B) Flor estaminada, C) Flor pistilada, D) Fruto. Basado en los ejemplares I. Rodríguez \& J.I. Calzada 286, 287 y 290. 
$\mathrm{km}$ de la desviación a El Porvenir, carretera MotozintlaHuixtla. $15^{\circ} 20.776$ 'N; $92^{\circ} 17.623^{\prime}$ W. Alt. 2059 m. 9/ 11/1999. I. Rodríguez \& J.I. Calzada 290. (hojas, flores estaminadas, flores pistiladas y frutos). (MEXU); Mpio. Motozintla de Mendoza. $11 \mathrm{Km}$ al SW de Motozintla, aproximadamente a $1 \mathrm{Km}$ antes de El Rosario, carretera Motozintla-Huixtla. $15^{\circ} 19^{\prime} \mathrm{N}$; $92^{\circ} 19^{\prime} \mathrm{W}$. Alt. 1800 m. 7/12 /1990. R. Lira \& A. Reyes 1283. (hojas, flores estaminadas y frutos). (MEXU); Mpio. Motozintla de Mendoza. $11 \mathrm{Km}$ al SW de Motozintla, camino a El Rosario. Alt. 1770 m. Veg. secundaria de bosque mesófilo. 28/10/1989. R. Lira, D. Sutton, R. Hampshire, J.C. Soto \& A. Reyes 949. (hojas, flores estaminadas, flores pistiladas y frutos). (BM, MEXU); Mpio. Motozintla de Mendoza. $2 \mathrm{Km}$ sobre el camino hacia el Cerro Mozotal y Siltepec (desviación sobre la carretera Motozintla-Huixtla). $15^{\circ} 23^{\prime} \mathrm{N}$; $92^{\circ} 15.5^{\prime}$ W. Alt. 2040 m. 7/12 /1990. R. Lira \& A. Reyes 1287. (hojas, flores estaminadas, flores pistiladas y frutos). (MEXU); Mpio. Motozintla de Mendoza. 4 $\mathrm{Km}$ above Tolimán on road to Motozintla. Alt. 1380 m. 22/11/1980. D.E. Breedlove \& F. Almeda 47655. (hojas, flores estaminadas, flores pistiladas y frutos). (MEXU); Mpio. Motozintla de Mendoza. Along Hwy. México 190, $10 \mathrm{Km}$ (by air) SW of Motozintla, E of Belisario Domínguez. $15^{\circ} 19^{\prime} \mathrm{N}$; 92 $20^{\circ} \mathrm{W}$. Alt. $1300 \mathrm{~m}$. 24/ 12/ 1985. T.C. Andres \& M. Nee 139. (hojas, flores estaminadas y frutos). (MEXU, MO, NY); Mpio. Zinacantán. Piedra Parada, aproximadamente $3 \mathrm{Km}$ al W de Chainatic, carretera Tuxtla Gutiérrez-San Cristóbal de las Casas. $16^{\circ} 45^{\prime} \mathrm{N}$; $92^{\circ} 48^{\prime} \mathrm{W}$. Alt. $1600 \mathrm{~m} .5 /$ 12 /1990. R. Lira \& A. Reyes 1258. (hojas, flores estaminadas, flores pistiladas y frutos). (MEXU). Oaxaca. Mpio. Huautla de Jiménez. Huautla de Jiménez, District of Teotitlán. 20/7/1938. R.E. Schultes \& B.P. Reko 220. (hojas, flores estaminadas y frutos). (GH); Mpio.
Huautla de Jiménez. Huautla, $2 \mathrm{Km}$ SW of market. $18^{\circ} 08^{\prime} \mathrm{N} ; 96^{\circ} 52^{\prime}$ 'W. Alt. 1400 m. 15/1/1984. S. Solheim \& S. Reisfield 1353. (hojas y frutos). (NY).

\section{Agradecimientos}

Los autores desean expresar su agradecimiento a los curadores de los herbarios mencionados en el trabajo por el préstamo de los materiales, al Dr. Fernando Chiang Cabrera (MEXU) por la elaboración de la diagnosis en latín de la nueva especie. La ilustración es obra del Biól. Julio César Flores Sánchez. Este trabajo se realizó gracias al apoyo del Herbario Nacional de México (MEXU), la beca de CONACyT número 92140 otorgada a la primera autora y al apoyo de la Comisión Nacional para el Conocimiento y Uso de la Biodiversidad, a través del proyecto Estado Actual y Fitogeografía de las Especies de la Familia Cucurbitaceae Endémicas a México (CONABIO-Q010), a cargo del segundo autor de 1998 a 2001.

\section{Literatura citada}

Jeffrey, C. 1990. Appendix: An Outline Clasification of the Cucurbitaceae. In: D.M. Bates,

R.W. Robinson \& C. Jeffrey (Eds.), Biology and Utilization of the Cucurbitaceae. Cornel University Press, Ithaca, NY. pp. 449-463.

Linneo, C. 1753. Species Plantarum. A Facsimile of the first edition. (1957). The Ray Society. London.

Martínez-Crovetto, R. 1964. Las Especies Argentinas del Género Sicyos (Cucurbitaceae). Bonplandia. 335-362.

Rodríguez-Arévalo, N.I. 2001. Revisión del Género Sicyos L. (Cucurbitaceae) en México. Tesis de Maestría en Ciencias. Facultad de Ciencias, Universidad Nacional Autónoma de México, México, D.F. 184 pp. 